Original

\title{
Natural Occurrence of Neoplastic Lesions in Young Sprague- Dawley Rats
}

\author{
Shinichiro Ikezaki ${ }^{1}$, Mizuho Takagi ${ }^{1}$, and Kazutoshi Tamura ${ }^{1}$ \\ ${ }^{1}$ Pathology Department, Gotemba Laboratory, Bozo Research Center Inc., 1284 Kamado, Gotemba, Shizuoka \\ 412-0039, Japan
}

\begin{abstract}
It is important for the assessment of toxicological effects of chemicals to know what kinds of neoplasms naturally occur in the early life of experimental animals. In the present study, we demonstrated spontaneous neoplasms in Sprague-Dawley rats used in 4-, 13- and 26-week toxicity studies conducted at Bozo Research Center in the last decade. The tumors, which were first observed in 19-week-old animals, included anterior adenoma of the pituitary, follicular cell adenocarcinoma and $\mathrm{C}$ cell adenoma of the thyroids, nephroblastoma of the kidneys, basal cell tumor of the skin and malignant lymphoma. Thereafter, hemangiosarcoma of the tongue, adenocarcinoma of the submandibular glands, histiocytic sarcoma of the spleen, oligodendroglioma of the brain and adenocarcinoma and fibroadenoma of the mammary glands were detected in 32-week-old animals. The incidences of mammary adenocarcinoma and pituitary anterior adenoma were higher than those of other tumors. The present results showed that the same tumors as reported in aged rats could also develop in younger rats. (DOI: 10.1293/tox.24.37; J Toxicol Pathol 2011; 24: 37-40)
\end{abstract}

Key words: spontaneous tumor, Sprague-Dawley rat, young rat

\section{Introduction}

In preclinical safety assessment of drugs and chemicals, animal studies are conducted. The results of histological examination are assessed from such viewpoints as dosedependency, histological peculiarity, correspondence to age and disclosed information concerning toxicity of the test compound. For neoplastic changes, historical control data are quite helpful in evaluating the results of studies; however, most published data have been collected from aged animals ${ }^{1-6}$. Therefore, it is sometimes difficult to make a decision about whether or not the lesions, especially proliferative ones, are compound-related when they are observed only in a high dose group with a low incidence in short-term toxicity studies.

Sprague-Dawley rats have been universally used in short-term toxicity studies, and they also have been the most frequently used experimental animals in our laboratory. There are several reports of general profiles of the occurrence of spontaneous tumors in aged Sprague-Dawley rats ${ }^{1-5}$, whereas the data available for younger Sprague-Dawley rats

(C)2011 The Japanese Society of Toxicologic Pathology

Received: 4 August 2010, Accepted: 25 October 2010

Mailing address: Shinichiro Ikezaki, Pathology Department, Gotemba Laboratory, Bozo Research Center Inc., 1284 Kamado, Gotemba, Shizuoka 412-0039, Japan

TEL: 81-550-82-9914 FAX: 81-550-82-9915

E-mail: ikezaki-shinichiro@bozo.co.jp are limited ${ }^{7}$. The situation is similar for other rat strains. It has recently been reported that the most common early tumor in both sexes of Han Wistar rats was malignant lymphoma $^{8}$.

Son et al. investigated the early occurrence of spontaneous tumors in Sprague-Dawley rats that died in the course of long-term carcinogenicity studies and revealed that the most common tumor in young Sprague-Dawley rats was adenoma of the pituitary gland, followed by mammary fibroadenoma and adenocarcinoma ${ }^{7}$. Their results are comparable to our data and are very useful in interpreting data recorded in short-term studies. However, the data from long-term studies do not always supply enough information for short-term studies, since most internal tumors are undetectable without necropsy.

To clarify which kinds of spontaneous neoplastic lesions occur first in Sprague-Dawley rats, we surveyed our historical data from 4-, 13- and 26-week toxicity studies conducted at Bozo Research Center Inc. in the last decade.

\section{Materials and Methods}

Data were obtained from Sprague-Dawley rats in the control groups in 4- (782 males and 770 females), 13(857 males and 878 females) and 26-week toxicity studies ( 842 males and 874 females) conducted at Bozo Research Center Inc. from 2000 to 2009, in which chemicals were given by various routes such as the oral, intravenous, sub- 
Table 1. Occurrence of Spontaneous Tumors in Control Sprague-Dawley Rats at 10 to 32 Weeks of Age

\begin{tabular}{|c|c|c|c|c|c|c|c|c|}
\hline \multirow[t]{3}{*}{ Organ/system } & \multirow[t]{3}{*}{ Tumor type } & \multirow{3}{*}{$\begin{array}{l}\text { Age (weeks old) } \\
\text { Sex } \\
\text { Number of animals }\end{array}$} & \multicolumn{2}{|c|}{10} & \multicolumn{2}{|c|}{19} & \multicolumn{2}{|c|}{32} \\
\hline & & & Male & Female & Male & Female & Male & Female \\
\hline & & & 782 & 770 & 857 & 878 & 842 & 874 \\
\hline Pituitary & Anterior adenoma & & - & - & - & $1(0.1)$ & $1(0.1)$ & $3(0.3)$ \\
\hline \multirow[t]{2}{*}{ Thyroid } & Follicular adenocarcinoma & & - & - & $1(0.1)$ & - & - & - \\
\hline & $\mathrm{C}$ cell adenoma & & - & - & - & $1(0.1)$ & - & - \\
\hline Tongue & Hemangiosarcoma & & - & - & - & - & $1(0.1)$ & - \\
\hline Submandibular gland & Adenocarcinoma & & - & - & - & - & $1(0.1)$ & - \\
\hline Spleen & Histiocytic sarcoma & & - & - & - & - & $1(0.1)$ & - \\
\hline Kidney & Nephroblastoma & & - & - & - & $1(0.1)$ & - & - \\
\hline Brain & Oligodendroglioma & & - & - & - & - & $1(0.1)$ & $2(0.2)$ \\
\hline Skin & Basal cell tumor & & - & - & $1(0.1)$ & - & - & - \\
\hline \multirow[t]{2}{*}{ Mammary gland } & Adenocarcinoma & & - & - & - & - & $1(0.1)$ & $6(0.7)^{\mathrm{a}}$ \\
\hline & Fibroadenoma & & - & - & - & - & - & $2(0.2)^{\mathrm{a}}$ \\
\hline Hemolymphoreticular system & Malignant lymphoma & & - & - & $1(0.1)$ & - & - & - \\
\hline \multirow{2}{*}{\multicolumn{2}{|c|}{$\begin{array}{l}\text { Number of tumor-bearing rats } \\
\text { Incidence of rats bearing tumors (\%) }\end{array}$}} & & 0 & 0 & 3 & 3 & 6 & 13 \\
\hline & & & 0 & 0 & 0.4 & 0.3 & 0.7 & 1.5 \\
\hline
\end{tabular}

Numbers in parentheses indicate incidence (\%). a The combined tumor incidence was $0.9 \%$.

cutaneous and dermal routes. The rats were obtained from Charles River Laboratories (Kanagawa and Shiga, Japan), and housed individually in an environmentally controlled animal room (temperature of $23 \pm 3{ }^{\circ} \mathrm{C}$, relative humidity of $55 \pm 20 \%$ and $12 \mathrm{~h} / 12 \mathrm{~h}$ light/dark cycle) and fed a commercial diet (CRF-1 and CR-LPF, Oriental Yeast Co., Ltd., Tokyo, Japan) and tap water ad libitum during the examination period.

The animals started the studies at 6 weeks of age and were euthanized under ether anesthesia at 10,19 or 32 weeks of age after a 4-, 13- or 26-week treatment, respectively. At necropsy, whole organs were subjected to a careful gross examination, and all abnormal findings were recorded. For nodular lesions suggesting neoplastic changes, the size, number and topographic information were also recorded. After necropsy, the brain, spinal cord, sciatic nerves, eyeballs, Harderian glands, pituitary, thyroids, parathyroids, adrenals, thymus, spleen, submandibular lymph nodes, mesenteric lymph nodes, heart, thoracic aorta, trachea, lungs (including bronchus), tongue, esophagus, stomach, duodenum, jejunum, ileum, cecum, colon, rectum, submandibular glands, sublingual glands, liver, pancreas, kidneys, urinary bladder, testes, epididymides, prostate, seminal vesicles, ovaries, uterus, vagina, mammary glands (inguinal), sternum (including bone marrow), femurs (including knee joint and bone marrow), femoral muscles, skin (inguinal) and other gross lesions were removed. The tissues excluding the eyes, optic nerve and testes were preserved in $10 \%$ phosphate-buffered formalin. The eyes and optic nerves were fixed in phosphate buffered 3\% glutaraldehyde/2.5\% formalin or Davidson's fluid, and the testes were fixed in Bouin's solution. After fixation, the tissues were embedded in paraffin, sectioned and stained with hematoxylin and eosin (H.E.). All studies for this survey were conducted in accordance with the Guide for Animal Experimentation of Bozo Research Center Inc. Data from dead animals was excluded from this survey because no neoplasms were seen.

\section{Results}

\section{Occurrence of tumors in rats at 10 weeks of age (Table 1)}

No neoplastic lesions were seen in any animals.

\section{Occurrence of tumors in rats at 19 weeks of age (Table 1)}

The tumors first identified occurred in 3 males and 3 females at 19 weeks of age. None of these tumor-bearing animals showed a moribund state. In males, follicular adenocarcinoma of the thyroids, basal cell tumor of the skin and malignant lymphoma were observed, and the incidence was $0.1 \%$ for each tumor. In females, anterior adenoma of the pituitary, $\mathrm{C}$ cell adenoma of the thyroids and nephroblastoma in the kidneys were observed, and the incidence was $0.1 \%$ for each tumor. The incidence of tumor-bearing rats was $0.4 \%$ for males and $0.3 \%$ for females.

\section{Occurrence of tumors in rats at 32 weeks of age (Table 1)}

In 32-week-old animals, 6 males and 13 females had tumors. None of these tumor-bearing animals showed a moribund state. In males, the tumors observed first at 32 weeks of age were anterior adenoma of the pituitary, hemangiosarcoma in the tongue, adenocarcinoma in the submandibular glands, histiocytic sarcoma in the spleen, oligodendroglioma in the brain and adenocarcinoma in the mammary glands, and the incidence was $0.1 \%$ for each tumor. In females, the tumors observed first at 32 weeks of age were oligodendro- 
Table 2. Macroscopic Lesions Correlating with Tumors Found in Control Sprague-Dawley Rats at 19 to 32 Weeks of Age

\begin{tabular}{|c|c|c|c|c|c|c|c|c|}
\hline \multirow[t]{3}{*}{ Organ/system } & \multirow[t]{3}{*}{ Tumor type } & \multirow[t]{3}{*}{$\begin{array}{l}\text { Total no. } \\
\text { of cases }\end{array}$} & \multirow{3}{*}{$\begin{array}{l}\text { Age } \\
\text { Sex } \\
\text { Rats } \\
\text { examined }\end{array}$} & \multicolumn{2}{|c|}{$\begin{array}{c}19 \\
\text { (weeks old) }\end{array}$} & \multicolumn{2}{|c|}{$\begin{array}{c}32 \\
\text { (weeks old) }\end{array}$} & \multirow{3}{*}{$\begin{array}{l}\text { Ratio of } \\
\text { tumors with } \\
\text { macroscopic } \\
\text { lesions }\end{array}$} \\
\hline & & & & Male & Female & Male & Female & \\
\hline & & & & 857 & 878 & 842 & 874 & \\
\hline Pituitary & Anterior adenoma & 5 & & - & $\mathrm{N}$ & $\begin{array}{l}\text { Dark red } \\
\text { focus }\end{array}$ & N, N, Nodule & $2 / 5$ \\
\hline \multirow[t]{2}{*}{ Thyroid } & Follicular adenocarcinoma & 1 & & Large & - & - & - & $1 / 1$ \\
\hline & $\mathrm{C}$ cell adenoma & 1 & & - & $\mathrm{N}$ & - & - & $0 / 1$ \\
\hline Tongue & Hemangiosarcoma & 1 & & - & - & Nodule & - & $1 / 1$ \\
\hline Submandibular gland & Adenocarcinoma & 1 & & - & - & Nodule & - & $1 / 1$ \\
\hline Spleen & Histiocytic sarcoma & 1 & & - & - & Nodule & - & $1 / 1$ \\
\hline Kidney & Nephroblastoma & 1 & & - & Nodule & - & - & $1 / 1$ \\
\hline Brain & Oligodendroglioma & 3 & & - & - & $\mathrm{N}$ & $\mathrm{N}, \mathrm{N}$ & $0 / 3$ \\
\hline Skin & Basal cell tumor & 1 & & Nodule & - & - & - & $1 / 1$ \\
\hline \multirow[t]{2}{*}{ Mammary gland } & Adenocarcinoma & 7 & & - & - & Nodule & $\begin{array}{l}\mathrm{N}, \mathrm{N}, \mathrm{N}, \mathrm{N} \\
\text { Nodule, } \\
\text { Nodule }\end{array}$ & $3 / 7$ \\
\hline & Fibroadenoma & 2 & & - & - & - & N, Nodule & $1 / 2$ \\
\hline Hemolymphoreticular system & Malignant lymphoma & 1 & & Large $^{\mathrm{a}}$ & - & - & - & $1 / 1$ \\
\hline
\end{tabular}

Individual findings or $\mathrm{N}$ (no macroscopic lesions in correlation with tumor) are presented as macroscopic lesions. a Lymph nodes (cervical, axillary, pancreatic), liver and spleen.

glioma in the brain and adenocarcinoma and fibroadenoma of the mammary glands, and the incidence was $0.2,0.7$ and $0.2 \%$, respectively. The female mammary gland was the organ that showed the highest tumor incidence (overall incidence $=0.9 \%$ ), and mammary adenocarcinoma was the spontaneous tumor showing the highest incidence $(0.7 \%)$. As compared with the 19-week-old females, the incidence of pituitary anterior adenoma was slightly elevated in the 32-week-old animals. In addition, the incidence of tumorbearing rats was slightly increased in both sexes.

\section{Macroscopic lesions related to tumors found in rats (Table 2)}

In both the 19- and 32-week-old animals, many types of tumors, except for C-cell adenoma and oligodendroglioma, were identified macroscopically by nodule formation, enlargement and discolored focus. Specifically, $52 \%$ of the tumor-bearing cases $(25$ cases) could be judged to have a tumor macroscopically; in other words, about half the cases were first found by microscopic examination.

\section{Discussion}

In the present study, we surveyed a total of 2481 male and 2522 female Sprague-Dawley rats from 10 to 32 weeks of age. In males, follicular adenocarcinoma of the thyroids, basal cell tumor of the skin and malignant lymphoma were identified at 19 weeks of age, and anterior adenoma in the pituitary, hemangiosarcoma in the tongue, adenocarcinoma in the submandibular glands, histiocytic sarcoma in the spleen, oligodendroglioma in the brain and adenocarcinoma in the mammary glands were first observed at 32 weeks of age. Thus, the variety of tumors tended to increase with age, but each tumor was observed only in one animal. These results suggest that the occurrence of such tumors in males of this age may not be age related. On the other hand, in females, anterior adenoma in the pituitary, $\mathrm{C}$ cell adenoma in the thyroids and nephroblastoma in the kidneys were identified at 19 weeks of age, and oligodendroglioma in the brain and adenocarcinoma and fibroadenoma of the mammary glands were first observed at 32 weeks of age. At 32 weeks of age, mammary tumors including fibroadenoma and adenocarcinoma developed in several animals, and the incidence of pituitary anterior adenoma was slightly higher than that at 19 weeks of age. These data suggest that the occurrence of mammary and pituitary tumors in females may be age related, since it is well known that such tumors spontaneously occur at a high incidence in aged rats of this strain ${ }^{1-5}$.

As mentioned in the introduction, Son et al. investigated tumors that occurred in all dead animals during the first 50 weeks of carcinogenicity studies in order to determine the profile of tumor occurrence in young Sprague-Dawley rats ${ }^{7}$. The data obtained from the dead animals are unsuitable for direct comparison with our data from the viewpoint of tumor incidence, because our data were based on animals euthanized as scheduled after a certain period, and their data was for animals that died at several time points during experiments. Although there was a difference in study design, both our results and those of Son et al..$^{7}$ demonstrated that mammary and pituitary tumors were relatively com- 
mon tumors in Sprague-Dawley rats. This also suggests that mammary and pituitary tumors may commonly occur in young as well as aged Sprague-Dawley rats.

Useful information on tumor incidence obtained from short-term studies is limited in every rat strain. In Han Wistar rats, it was recently reported that no tumors were found in short-term studies, including 2-, 4-, 13- and 26-week studies, using a total of 895 males and 895 females $^{8}$. This report shows that tumor occurrence in Han Wistar rats is lower than that in Sprague-Dawley rats at a young age. Such a strain difference in the occurrence of spontaneous tumors between young Han Wistar and Sprague-Dawley rats could also apply to the aged rats of these two strains.

It is interesting that the incidence of oligodendroglioma was relatively high in animals at 32 weeks of age because this type of tumor is thought to rarely occur, even in aged rats. Specifically, the incidence of oligodendroglioma was $0 \%$ in our historical control data for long-term studies using Sprague-Dawley rats from 2003 to 2007, and it was also reported to be $0 \%$ or nearly $0 \%$ in past studies making use of other historical data ${ }^{1-5}$. Three cases of oligodendroglioma were detected in one rat each purchased in 2004, 2005 and 2007 from the same breeding site. Therefore, whether or not a genetic factor influenced the occurrence of this tumor is unclear.

Adenocarcinoma of the salivary glands, a very rare tumor even in aged rats 2,4 , was seen in one 32-week-old male rat, indicating the difficulty of showing an age-related tendency in the occurrence of very rare tumors.

To evaluate the microscopic findings in toxicity studies correctly, careful reference to historical control data is very important. In this regard, the present study will be useful in evaluating whether a tumor is incidental or not when an unexpected tumor is found in young rats. However, we should take notice of the fact that tumor development is affected by various factors such as diet, husbandry, environmental conditions, housing style and source of animals.

In conclusion, the present study suggests that spontaneous mammary and pituitary tumors that are commonly observed in aged female rats begin to develop at or before 32 weeks of age and that tumors unrelated to age may sporadi- cally occur in various tissues.

Acknowledgments: The authors gratefully acknowledge Dr. Kunio Doi, Professor Emeritus of the University of Tokyo, for critical review of the manuscript. We are also grateful to Mr. Pete Aughton, ITR Laboratories Canada Inc., for proofreading.

\section{References}

1. Kaspareit J, and Rittinghausen S. Spontaneous neoplastic lesions in Harlan Sprague-Dawley rats. Exp Toxicol Pathol. 51: 105-107. 1999.[Medline]

2. Chandra M, Riley MG, and Johnson DE. Spontaneous neoplasms in aged Sprague-Dawley rats. Arch Toxicol. 66: 496-502. 1992.[Medline] [CrossRef]

3. Nakazawa M, Tawaratani T, Uchimoto H, Kawaminami A, Ueda M, Ueda A, Shinoda Y, Iwakura K, Kura K, and Sumi N. Spontaneous neoplastic lesions in aged Sprague-Dawley rats. Exp Anim. 50: 99-103. 2001.[Medline] [CrossRef]

4. McMartin DN, Sahota PS, Gunson DE, Hsu HH, and Spaet RH. Neoplasms and related proliferative lesions in control Sprague-Dawley rats from carcinogenicity studies. Historical data and diagnostic considerations. Toxicol Pathol. 20: 212-225. 1992.[Medline] [CrossRef]

5. Baldrick P. Carcinogenicity evaluation: comparison of tumor data from dual control groups in the Sprague-Dawley rat. Toxicol Pathol. 33: 283-291. 2005.[Medline]

6. Maita K, Hirano M, Harada T, Mitsumori K, Yoshida A, Takahashi K, Nakashima N, Kitazawa T, Enomoto A, Inui K, and Shirasu Y. Mortality, major cause of moribundity, and spontaneous tumors in CD-1 mice. Toxicol Pathol. 16: 340-349. 1988.[Medline] [CrossRef]

7. Son WC, and Gopinath C. Early occurrence of spontaneous tumors in CD-1 mice and Sprague-Dawley rats. Toxicol Pathol. 32: 371-374. 2004.[Medline] [CrossRef]

8. Son WC, Bell D, Taylor I, and Mowat V. Profile of early occurring spontaneous tumors in Han Wistar rats. Toxicol Pathol. 38: 292-296. 2010.[Medline] [CrossRef] 